\title{
Impactos físicos e emocionais na saúde do cuidador informal de pacientes com
}

\section{doença de Alzheimer}

Physical and emotional impacts on the health care of informal caregivers of patients with

\author{
Alzheimer's disease
}

Impactos físicos y emocionales em la atención médica de los cuidadores informales de pacientes com enfermedad de Alzheimer

Pedro Henrique de Souza ORCID: https://orcid.org/0000-0002-4239-9211 Universidade Estadual de Mato Grosso do Sul, Brasil E-mail: pedrowiskiii@ outlook.com

Priscylla Frazão Rodrigues

ORCID: https://orcid.org/0000-0001-7580-516X Universidade Federal do Delta do Parnaíba, Brasil E-mail: priscyllafrazao1995@ gmail.com

Rafaella Silva Alcantara

ORCID: https://orcid.org/0000-0002-0741-7883 Universidade Federal de Alagoas, Brasil E-mail: rafaella.salcantara@gmail.com

Robério Araújo de Carvalho ORCID: https://orcid.org/0000-0002-9422-0172 Universidade Federal do Delta do Parnaíba, Brasil E-mail: roberiocarvalho1992@gmail.com

Jhonantas Henrique Brito Santos ORCID: https://orcid.org/0000-0001-9790-6041 Universidade Federal do Delta do Parnaíba, Brasil

E-mail: Jhonantas.brito@gmail.com

Mauro Mendes Pinheiro Machado ORCID: https://orcid.org/0000-0002-3165-6120 Universidade Federal do Delta do Parnaíba, Brasil E-mail: mauromp@ hotmail.com

\begin{abstract}
Resumo
A doença de Alzheimer afeta de forma bastante importante as atividades diárias do doente e das pessoas responsáveis pelo seu cuidado. Desta forma, haja vista o grande desgaste físico e emocional dos cuidadores, o objetivo deste trabalho foi analisar como o cuidado dispensado aos pacientes com Alzheimer pode impactar física e emocionalmente os cuidadores. Trata-se de uma revisão integrativa de literatura, aonde foram utilizadas as bases de dados Scielo e PubMED, e os descritores "impactos físicos", "impactos emocionais", "cuidadores”, “doença de Alzheimer" e "familiares", em diferentes combinações. Foram incluídos artigos publicados entre 2010 e 2020, nos idiomas inglês, espanhol, português e francês. Observou-se que a maioria dos cuidadores de pacientes demenciados são mulheres, em geral, familiares do doente. Além disso, com o progresso da doença, o esgotamento físico e emocional dos cuidadores tende a aumentar, provocando estresse exacerbado, aparecimento de doenças crônicas, ansiedade, abdicação de atividades prazerosas ao cuidador, depressão e sentimentos de solidão e abandono. Dessa forma, visualizou-se que o processo de cuidado dos pacientes com Alzheimer é bastante complexo, sobretudo nos estágios mais avançados da doença, provocando abdicação das vontades do cuidador e impactos físicos e emocionais negativos na sua saúde e qualidade de vida.
\end{abstract}

Palavras-chave: Doença de Alzheimer; Cuidadores; Qualidade de vida; Neurologia.

\begin{abstract}
Alzheimer's disease significantly affects the daily activities of the patient and the people responsible for his/her care. Thus, given the great physical and emotional wear of caregivers, the objective of this study was to analyze how the care provided to patients with Alzheimer's can physically and emotionally impact caregivers. This is an integrative literature review, using the Scielo and PubMED databases, and the descriptors "physical impacts", "emotional impacts", "caregivers", "Alzheimer's disease" and "family members", in different combinations. Articles published between 2010 and 2020, in English, Spanish, Portuguese and French were included. It was observed that most
\end{abstract}


caregivers of demented patients are women, in general, family members of the patient. In addition, with de progress of the disease, the physical and emotional exhaustion of caregivers tends to increase, causing exacerbated stress, the onset of chronic diseases, anxiety, abdications of pleasurable activities for the caregiver, depression and feelings of loneliness and abandonment. Thus, it was seen that the care process of patients with Alzheimer's is quite complex, especially in the more advanced stages of the disease, causing abdications od the caregiver's wishes and negative physical and emotional impacts on their health and quality of life.

Keywords: Alzheimer disease; Caregivers; Quality of life; Neurology.

\section{Resumen}

La enfermedad de Alzheimer afecta significativamente las actividades diarias del paciente y las personas responsables de su cuidado. Así, dado el gran desgaste físico y emocional de los cuidadores, el objetivo de este estudio fue analizar cómo la atención brindada a los pacientes con Alzheimer puede impactar física y emocionalmente a los cuidadores. Se trata de una revisión integradora de la literatura, utilizando las bases de datos Scielo y PubMED, y los descriptores "impactos físicos", "impactos emocionales", "cuidadores", "enfermedad de Alzheimer" y "miembros de la familia", en diferentes combinaciones. Se incluyeron artículos publicados entre 2010 y 2020, en inglés, español, portugués y francés. Se observó que la mayoría de los cuidadores de pacientes dementes son mujeres, en general familiares del paciente. Además, con el avance de la enfermedad tiende a incrementarse el agotamiento físico y emocional de los cuidadores, provocando un agravamiento del estrés, aparición de enfermedades crónicas, ansiedad, abdicación de actividades placenteras para el cuidador, depresión y sentimientos de soledad y abandono. Así, se vio que el proceso de atención de los pacientes con Alzheimer es bastante complejo, especialmente en las etapas más avanzadas de la enfermedad, provocando abdicación de los deseos del cuidador e impactos físicos y emocionales negativos en su salud y calidad de vida.

Palabras clave: Enfermedad de Alzheimer; Cuidadores; Calidad de vida; Neurología.

\section{Introdução}

O aumento da proporção de idosos em relação a outras faixas etárias reflete o crescimento da expectativa de vida e uma consequente mudança no cenário epidemiológico das patologias, uma vez que passa a ocorrer o predomínio das doenças crônico-degenerativas e não transmissíveis. Nesse cenário, destaca-se a demência, que afeta cerca de 46 milhões de pessoas no mundo e aumenta, consideravelmente, o número de idosos vulneráveis (Ponce et al., 2011; Santos \& Pavarini, 2012; Farina et al., 2017).

As síndromes demenciais apresentam-se por um declínio de diversas funções corticais superiores, caracterizando-se por irreversíveis comprometimentos de função intelectual, emocional e comportamental (Perdomo \&, Rodríguez, 2010; Terum et al., 2017). A doença de Alzheimer (DA) constitui o tipo mais frequente de demência (70-80\% dos casos), denominada, atualmente, como a "epidemia do século" e incluída, pela Organização Mundial de Saúde (OMS), como uma das afecções prioritárias em relação à Saúde Mental (Perdomo \& Rodríguez, 2010). Essa patologia, por apresentar deterioração constante da capacidade de realizar atividades rotineiras, tem um impacto significativo na vida do paciente, visto que se torna mais dependente do cuidador/família (Perdomo \& Rodríguez, 2010; Terum et al., 2017).

Tal realidade, além de gerar conflitos se não for tratada adequadamente, impõe mudanças na rotina e na estrutura familiar, já que membros da família são encarregados de assumir, na maioria das vezes, o papel de cuidador (Ponce et al., 2011). O grupo dos cuidadores inclui aqueles com trabalho formal, que recebem para cuidar profissionalmente e podem ou não ter preparo técnico para o exercício da função (Ponce et al., 2011; Córdoba \& Aparicio, 2014), e aqueles cujo trabalho é informal, ou seja, não é remunerado e a carga horária está diretamente relacionada ao grau de parentesco entre o paciente e o cuidador. Esse grupo abrange, geralmente, familiares ou algum membro da comunidade que tomam para si a responsabilidade de cuidar (Ponce et al., 2011; Petrovic \& Gaggioli, 2020).

As famílias de pessoas com DA carregam um fardo muito pesado ao receberem o diagnóstico da doença de seu familiar, precisando reajustar recursos humanos e financeiros para cuidar dele. Muitas vezes, a falta de conhecimento acerca da doença, além do escasso apoio afetivo e técnico para lidar com a progressão da enfermidade, comprometem a saúde física e mental dos cuidadores, o que leva a transtornos emocionais, comportamentais e neuropsiquiátricos (Araújo, Oliveira, \& 
Pereira, 2012). Essa falta de suporte técnico e emocional evidencia a relevância de novos estudos sobre o tema, uma vez que cuidadores não treinados para o manejo da doença têm maior probabilidade de lidar com altos níveis de estresse e sofrer de distúrbios de saúde mental (Córdoba \& Aparicio, 2014).

Ademais, pesquisas apontam que o cuidado é realizado, preponderantemente, por mulheres, cônjuges, entre a quinta e sexta década de vida e nível de escolaridade que varia de baixo a médio. Essa realidade implica diretamente em uma elevada sobrecarga da cuidadora que também assume outros papéis sociais (Charlesworth et al., 2016; Marins, Hansel, \& Silva, 2016; Manzini \& Vale, 2020).

Apesar de muitos considerarem o ato de cuidar uma forma de retribuição moral ao familiar com DA, uma ação ética em nível pessoal e social, o grupo dos cuidadores, em comparação com a população em geral, apresenta sintomas ansiosos e depressivos, além da maior probabilidade de sofrer alterações em seus sistemas cardiovascular e imunológico (Córdoba \& Aparicio, 2014). Ademais, o cuidador lida com sentimentos ambivalentes em relação ao seu familiar com DA, como culpa, raiva e medo, experimentando a sensação que sua vida mudou desfavoravelmente devido à atividade de cuidado que ele agora realiza (Silva, Passos, \& Barreto, 2012).

Os prestadores de cuidados à pacientes com DA são classificados como grupo de alto risco para suicídio, e esse risco não parece desaparecer após a morte ou institucionalização do paciente (Kishita, Hammond, Dietrich, \& Mioshi, 2018), sendo este fato um alerta para os impactos permanentes do ato de cuidar na vida dessas pessoas, o que coaduna com a relevância dos estudos e intervenções neste grupo. Nessa perspectiva, objetiva-se descrever os principais impactos físicos e emocionais que interferem negativamente na qualidade de vida dos cuidadores informais de pacientes com Alzheimer.

\section{Metodologia}

O presente artigo estrutura-se como uma revisão integrativa da literatura, com síntese qualitativa, definida como uma agregação de dados coletados de bases secundárias através de levantamento bibliográfico. Este processo tem como intenção sintetizar as evidências disponíveis por meio de buscas e avaliações críticas, a fim de promover uma melhor compreensão de um determinado assunto (Souza, Silva, \& Carvalho, 2010). Para esta revisão, foram utilizadas como bases de dados as plataformas Scientific Electronic Library Online (SciELO) e PubMED/MEDLINE, pautando-se nos descritores em saúde "impactos físicos", "impactos emocionais", "cuidadores", "doença de Alzheimer" e "familiares", sendo utilizados em inglês e português. Os descritores foram utilizados em diferentes ordens de combinação, usando o operador booleano aditivo restritivo (“AND”) em ambas as plataformas.

Os artigos foram selecionados a partir de critérios de inclusão, os quais publicados entre 2010 e 2020, nos idiomas inglês, espanhol, português e francês. Além disso, foram selecionados trabalhos de artigos de revisão, ensaios clínicos e estudos qualitativos, ao passo que foram adotados como critérios de exclusão monografias, relatos de caso, capítulos de livro, estudos piloto, editoriais e publicações anteriores ao ano de 2010. Foram encontrados, a partir dessa busca, 111 artigos, sendo 52 no SciELO e 59 no PubMED/MEDLINE.

Após essa primeira análise, os artigos foram separados de forma homogênea para avaliação do título e resumo (abstract/resumen) por todos os autores, sendo considerados adequados 30 artigos da plataforma SciELO e 28 estudos da base de dados PubMED/MEDLINE, os quais abordavam direta ou indiretamente a doença de Alzheimer, o papel do cuidador e as consequências do processo de cuidado à saúde do cuidador.

Por fim, após essa segunda seleção, dividiu-se novamente os artigos, no intuito de avaliar o texto completo. Todos os trabalhos passaram pela leitura crítica dos autores, sendo que os estudos com abordagem distinta da proposta inicial, como a descrição de outras síndromes demenciais, tratamento de aspectos clínicos da doença de Alzheimer e formas de intervenção na 
saúde do paciente em neurodegeneração foram excluídos. Com isso, outros 38 estudos foram desconsiderados, restando 20 artigos incluídos nessa revisão (Figura 1).

Figura 1: Fluxograma da metodologia de busca dos estudos.

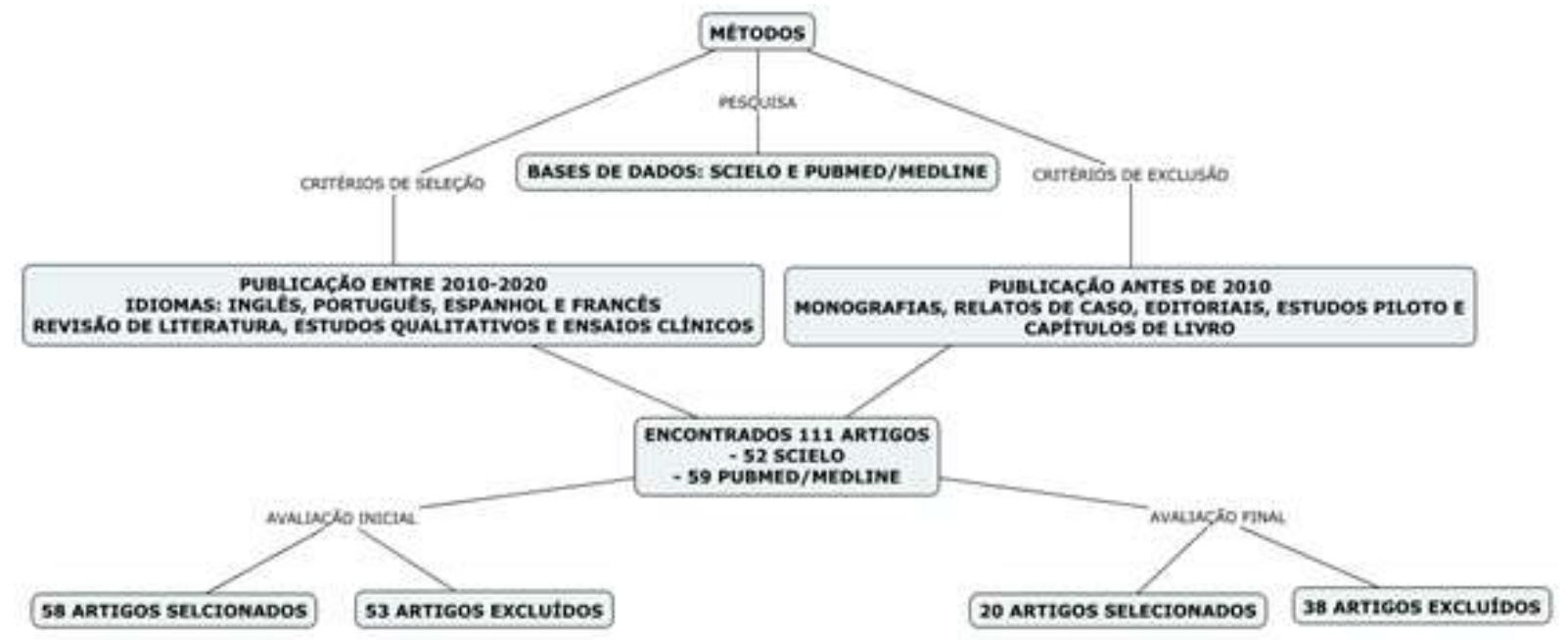

Fonte: Autores.

\section{Resultados e Discussão}

\subsection{Caracterização dos cuidadores de pacientes com Alzheimer}

Os cuidadores de indivíduos acometidos pela doença de Alzheimer (DA) são, em sua maioria, compostos por pessoas do sexo feminino, no qual se percebe preponderantemente uma relação de parentesco existente entre o cuidador e o doente, sendo notada a predominância de cônjuges e filhas. Observa-se que esse fator tem relação direta com a sobrecarga do cuidador, visto que além da responsabilidade de cuidado do familiar idoso, a mulher cuidadora tem uma variedade de papéis sociais como o de mãe, esposa, dona de casa e trabalho profissional, tendo efeitos negativos, não apenas na saúde física, mas também na mental do cuidador (Araújo et al., 2012; Marins et al., 2016; Manzini \& Vale, 2020). O fato de a DA ser neurodegenerativa e atingir em alto grau os aspectos sociocomportamentais e de autogerenciamento, além dos pacientes possuírem idade avançada, levam a um maior envolvimento da família na abordagem, o que explica a grande maioria de cuidados informais existentes (Gratao et al., 2010; Perdomo, Poey, \& Oca, 2012; Terum et al., 2017).

Em um estudo realizado em Belo Horizonte, capital de Minas Gerais, no Brasil, identificou-se que mais da metade das cuidadoras entrevistadas não se dedicavam integralmente ao ato de cuidar do paciente demenciado, haja vista que possuíam um vínculo empregatício externo (Silva et al., 2012). Em contraponto, outros estudos realizados no Brasil revelam que quase 70\% dos cuidadores informais despendem serviço integral ao doente, sendo que esse fato está intimamente relacionado ao grau de evolução do enfermo, uma vez que quanto mais avançada estiver a doença, mais horas de cuidados serão necessárias. Além disso, deve-se atentar ainda sobre o quanto a dedicação aos cuidados interferem nas outras atividades do cotidiano desses indivíduos, pois conforme os artigos, embora existam relatos de práticas de lazer nas horas vagas, o que contribui com o bemestar, o mesmo não se aplica a prática de atividades físicas regulares, fato que favorece o risco de morbidades cardiovasculares nesse grupo (Araújo et al., 2012; Petrovic \& Gaggioli, 2020).

Conforme Marins et al. (2016), outro aspecto visualizado no perfil sociodemográfico dos cuidadores de pacientes com DA, relaciona-se ao grau de instrução desses indivíduos, sendo notada a existência de poucos casos de analfabetos, além de observar-se uma porcentagem considerável de prestadores de cuidados com acesso ao nível superior, mesmo dentre aqueles que prestam cuidados informais. Com relação ao conhecimento acerca da doença de Alzheimer, Gratao et al. (2010) afirmaram 
que mais de $60 \%$ dos cuidadores assumem ter sapiência suficiente para realizar as atividades que envolvem o cotidiano dos doentes. No entanto, ainda referem que menos de $20 \%$ dos entrevistados tiveram acesso a algum tipo de curso ou orientação formal sobre a temática.

No que diz respeito ao conhecimento sobre o manejo do paciente e dificuldades inclusas no papel de cuidar de um enfermo demenciado, a existência de poucas redes de apoio com aparato técnico e de suporte à saúde, constitui-se como um grande desafio enfrentado pelos cuidadores informais, uma vez que a maioria deles nunca prestou esse papel de cuidar ou compartilhar o cuidado. Além disso, a exaustão do trabalho somada às reações adversas do paciente com Alzheimer frente à figura do cuidador pode implicar em consequências físicas e emocionais em curto e longo prazos sobre esses indivíduos, como doenças crônicas e sofrimento psíquico (Ponce et al. 2011; Córdoba \& Aparício, 2014; Petrovic \& Gaggioli, 2020).

Ademais, outro ponto importante a ser destacado, refere-se ao fato de o cuidado aos pacientes em neurodegeneração ser realizado, predominantemente, por apenas uma pessoa da família. Isso é influenciado pelo fato de que um número significativo de cuidadores já convivia previamente com o enfermo há muitos anos. Por conta disso, esses familiares acabam assumindo tarefas árduas, mesmo que de forma inexperiente. Todavia, essa realidade evidencia sentimentos de solidão e revela a necessidade que o cuidador tem de receber apoio familiar para evitar, ou pelo menos reduzir, a sobrecarga trazida pelo trabalho (Ponce et al., 2011; Araújo et al., 2012).

\subsection{Impactos físicos sobre os cuidadores dos pacientes com Alzheimer}

No momento em que se estabelecem os cuidados a um paciente com doença de Alzheimer, observam-se que ocorrem mudanças significativas no cotidiano dos cuidadores, principalmente quando esses são familiares. As inúmeras demandas do enfermo em neurodegeneração resultam em esgotamento físico e mental. Com o progresso da doença, essa situação tende a se agravar, uma vez que as atividades vão ficando mais complexas e o idoso fica cada vez mais dependente. Além disso, quando o cuidador assume a missão individual de cuidar do doente, sem tempo adequado de intervalo ou descanso, situações de desgaste e sobrecarga ocorrem frequentemente (Araújo et al., 2012; Marins et al., 2016).

Os impactos físicos para com a saúde do cuidador, derivados do desgaste advindo das atividades diárias não se limitam apenas a algias ou fadiga muscular, haja vista que podem comprometer uma série de componentes do corpo, além de alterar os níveis metabólicos de hormônios e substâncias, as quais em desequilíbrio podem acarretar doenças crônicas. O estresse persistente, por exemplo, resulta em uma secreção elevada de cortisol diurno pelas adrenais, fato que quando duradouro, pode levar à queda de atividade imunológica ou outros acometimentos endócrinos que podem colaborar para o surgimento de doenças agudas ou crônicas (Aboulafia-Brakha, Suchecki, Gouveia-Paulino, Nitrini, \& Ptak, 2014). Segundo Terum et al. (2017), os sintomas dos pacientes com doença de Alzheimer que mais impactam nos níveis de estresse dos cuidadores são a irritabilidade, agressividade, delirium, ansiedade, apatia, assim como distúrbios de sono, sendo que tais fatores podem gerar cargas diferentes e dependem da progressão da doença de base.

Além disso, como descrito por Moore et al. (2013), os elevados níveis de estresse cotidianos dos cuidadores podem contribuir para o aumento da concentração plasmática da interleucina-6 (IL-6), uma citocina pró-inflamatória, com atuação tanto na resposta imune inata quanto na adaptativa. Ela é sintetizada por monócitos, células endoteliais, fibroblastos e outras células, em resposta a microrganismos ou outras citocinas, como a IL-1. Essa molécula apresenta uma associação positiva com instabilidades coronárias e aterosclerose, de modo que sua elevação está relacionada com infartos agudos do miocárdio (IAM) não fatais. Além disso, a IL-6 interfere na síntese de insulina pelas células beta-pancreáticas, podendo contribuir para o desenvolvimento de hiperglicemia ou diabetes entre os cuidadores dos pacientes com Alzheimer.

Gratao et al. (2010), em um estudo abordando pacientes que sofrem com síndromes demenciais, em especial o Alzheimer, doença que correspondeu a mais de 50\% dos sujeitos abordados na análise, revelaram que a principal consequência 
do processo de cuidar dos idosos em neurodegeneração é o cansaço físico. Esse ocorre, sobretudo, durante os momentos de higiene e alimentação do paciente. Essa manifestação pode ser responsável pela gênese ou agravamento de vários outros sintomas comuns nos cuidadores, como cefaleia, artralgia, lombalgia e insônia, que acabam piorando deveras a qualidade de vida desses indivíduos (Silva et al., 2012; Araújo et al., 2012).

Por fim, conforme Coelho et al. (2015) e Leite et al. (2017), observa-se que o principal achado patológico no que se refere à saúde física do cuidador de idosos com DA é a hipertensão arterial sistêmica (HAS), uma doença com alta prevalência no Brasil. No entanto, deve-se salientar que essa condição acomete principalmente os cuidadores com idade superior a 60 anos. A associação entre hipertensão arterial e um ritmo de vida extremamente exaustivo e estressante, típico desses indivíduos, assim como a inatividade física e a ausência de hábitos alimentares saudáveis, favorecem as inúmeras complicações da HAS, como os eventos cerebrovasculares e as síndromes coronarianas.

\subsection{Impactos emocionais sobre os cuidadores}

Os impactos emocionais e psíquicos sobre os cuidadores de pessoas com doença de Alzheimer são fenômenos bastante comuns. A alteração drástica do estilo de vida, associado com a falta de apoio de boa parte dos familiares para enfrentar as sobrecargas físicas e emocionais, faz com que aqueles indivíduos desenvolvam inúmeras alterações psicológicas, dentre as quais se destacam os elevados níveis de estresse, diretamente relacionados ao estágio da neurodegeneração, a ansiedade, o sentimento de solidão e abandono, e a depressão (Araújo et al., 2012; Kishita et al., 2018; Manzini \& Vale, 2020).

Em relação aos níveis de estresse, inúmeros estudos mostraram que ele costuma ser proporcional ao estágio da doença. Isto é, quanto mais avançada se encontra a DA, maior é o estresse dos cuidadores. Todavia, outros fatores como mudanças da qualidade de vida, abdicação dos prazeres, instabilidade financeira, confusões amorosas e raiva reprimida, contribuem efetivamente para esse fenômeno (Gratao et al., 2010; Manzini \& Vale, 2020). Outro ponto importante dentro desse quadro é o desconhecimento da doença pelo cuidador. Os sentimentos de impotência e vulnerabilidade diante do quadro demencial progressivo favorecem a elevação do estresse desses indivíduos, assim como de manifestações emocionais associadas, como a ansiedade (Manzini \& Vale, 2020). O estudo de Córdoba e Aparício (2014), realizado com 53 cuidadores informais e 62 formais, torna claro o impacto que a ausência de preparo e estrutura dos familiares para realizar a função de cuidador tem na saúde desse grupo e, consequentemente, no sistema de saúde. Tal fato é notório nos 73,5\% dos cuidadores informais identificados com algum tipo de sobrecarga, sendo 58,5\% com sobrecarga intensa, necessitando de assistência médica, contrastando com apenas 33,9\% dos cuidadores formais com sobrecarga intensa.

O processo de cuidar, conforme Cesário et al. (2017), requer uma ampla variedade de comprometimentos por parte do cuidador, destacando-se os aspectos físicos, psicológicos, espirituais e sociais. Esse dar-se, de forma integral, gera desde cansaço e esgotamento físicos, até diminuição da autoestima e subvalorização das próprias necessidades. Acompanhado disso, podem surgir preocupações acerca da herança da neurodegeneração e da possibilidade do acometimento demencial no futuro. Tal panorama, de forma lógica, tende a criar no cuidador um sentimento de angústia e despreparo para exercer o ofício, desembocando em manifestações psicológicas de graus variáveis.

Ao considerar as particularidades da doença de Alzheimer, percebe-se que o cuidado a ser oferecido ao enfermo varia deveras em relação a outros tipos de demência. O fato de o cuidador estar presente nos diversos aspectos da vida do paciente, seja nas atividades simples ou complexas, repercute diretamente na saúde mental dos indivíduos. Esse processo de cuidado tão intrínseco favorece a gênese de um sentimento de apropriação do cuidado, aonde os demais membros da família, por exemplo, que queiram auxiliar no manejo do doente, acabam sendo vistos pelos cuidadores, como incapazes de desempenhar quaisquer 
atividades. Essa monopolização dos deveres gera cuidadores heroicamente estressados, que indiretamente, minguam sua saúde física e emocional (Ponce et al., 2011; Mattos \& Kovács, 2020).

Em relação à depressão, uma alteração bastante comum no grupo descrito, sabe-se que os cuidadores de pacientes com demência apresentam um alto risco de morte por suicídio. Essa característica não parece se alterar com a morte ou institucionalização do familiar em neurodegeneração (Kishita et al., 2018). Segundo Andrade (2012), esse transtorno psicológico tende a estar presente desde o início do cuidado, devido a processos como o luto antecipado, a deterioração mental e física do doente, com repercussões no relacionamento familiar, o escasso tempo e o abandono das próprias necessidades. $\mathrm{O}$ desenvolvimento do quadro depressivo relaciona-se, ainda, com diminuição da efetividade do sistema imune e com a descompensação de doenças crônicas prévias, agravando a saúde do cuidador e o processo de cuidar.

Devido à concepção social da doença de Alzheimer e ao complexo caminho diagnóstico da doença, é comum que os cuidadores desenvolvam um sentimento de luto antecipatório. O mesmo pode ser definido como um processo de antecipação e elaboração da perda futura de um ente querido, não no aspecto concreto da perda, isto é, da morte em si, mas sim dos pensamentos individuais, atividades conjuntas e perda da identidade que vai ocorrendo ao longo do tempo, devido à neurodegeneração progressiva. Tal fenômeno pode se apresentar de diversas formas, em geral variando com o estágio da doença. As principais manifestações do luto antecipatório incluem a negação, o superenvolvimento, a raiva, a culpa e a aceitação. A identificação desse processo nas fases iniciais do cuidado é fundamental, visto que está associada com menores sentimentos de culpa, pena, angústia, impotência e frustração, por conseguinte, melhorando os aspectos psicológicos do cuidar (Mattos \& Kovács, 2020; Manzini \& Vale, 2020).

Atualmente, não há registros específicos sobre o quantitativo dos cuidadores de idosos acometidos por demência de Alzheimer. No entanto, o aumento expressivo da população idosa, conforme já expresso anteriormente, assim como a prevalência de patologias demenciais em idosos com mais de 65 anos, conduz ao processo de maior necessidade de cuidadores e percebe-se a predominância do cuidado informal a esses enfermos (Gratao et al., 2010; Ponce et al., 2011), sendo notório alguns fatores como o desconhecimento da doença, a ausência de apoio familiar, as inseguranças do cuidado, a instabilidade financeira, o processo de institucionalização do cuidado, entre outros, intrinsecamente responsáveis por repercussões físicas e emocionais sobre esses indivíduos, como estresse, distúrbios pressóricos, ansiedade e depressão, percebendo-se, portanto, o potencial comprometimento de forma integral da vida dos cuidadores, o que induz a necessidade de atenção à saúde desses indivíduos prestadores de assistência alheia (Gratao et al., 2013; Córdoba \& Aparicio, 2014; Kucmanski et al., 2016; Mattos \& Kovács, 2020).

Nesse sentido, tem-se que as intervenções mais efetivas dizem respeito ao campo da psicoeducação. Trata-se de uma metodologia que traz a abordagem psicológica do cuidador aliada a uma perspectiva educacional que, por meio de um acompanhamento contínuo, tem por objetivo promover o gerenciamento do estresse dos cuidadores, advindo do cotidiano exaustivo. Além disso, serve para prestar todas as informações cabíveis ao cuidador, auxiliando na melhor administração dos esforços repetitivos e servindo como uma forma de aconselhamento para um melhor bem-estar fora do exercício do cuidar, além da questão do treinamento em cuidados pessoais e apoio social, o qual refletirá positivamente na saúde mental e física desses indivíduos (Gonçalves-Pereira \& Sampaio, 2011; Cheng et al., 2012; Aboulafia-Brakha et al., 2014).

Dessa forma, a atenção primária está intimamente relacionada a essas práticas de psicoeducação, mostrando-se viável à atuação de uma equipe multiprofissional no sentido de desenvolver esses exercícios, amparados tanto por um Núcleo de Apoio à Saúde da família (NASF), como por uma Equipe de Saúde da Família (ESF), mas que pode vislumbrar, também, o apoio da Rede de Atenção Psicossocial (RAPS) em casos que necessitem de uma maior especialização por parte do serviço público de saúde (Vizzachi, Daspett, Cruz, \& Horta, 2015; Campos, Carvalho, Barham, Andrade, \& Giannini, 2019). 
Outras alternativas, além da abordagem profissional especializada, com médicos e psicólogos, por exemplo, incluem a religião, expressa por vários autores como uma ótima alternativa de manifestação da espiritualidade e desafogo emocional, além da valorização das atividades aprazíveis aos cuidadores, como prática de exercícios físicos e encontros com os amigos, que servem como mecanismos de melhora da qualidade de vida e do cuidado ao enfermo (Gratao et al., 2013; Córdoba \& Aparicio, 2014; Kucmanski et al., 2016; Mattos \& Kovács, 2020).

\section{Conclusão}

Evidenciou-se que, na maioria dos casos, os cuidados dispensados aos pacientes com DA recaem, principalmente, sobre entes familiares do sexo feminino e com graus de instrução elevados. Este conhecimento favorece o oferecimento de cuidados satisfatórios a estes pacientes, porém com o decorrer do período de dispensa de cuidado e devido à carga de trabalho empregada no cuidado de uma pessoa acometida de Alzheimer, os impactos acabam por afetar, tanto física como psicologicamente as pessoas que assumem os serviços principais relacionados aos doentes, ocasionando o adoecimento do cuidador e refletindo diretamente nos cuidados dispensados.

Entre as principais afecções físicas, observou-se o esgotamento ocasionado pela sobrecarga de serviços relacionados ao ato de cuidar da pessoa com DA, que não se resumem a apenas algias ou fadigas, mas que influem e alteram o metabolismo do cuidador e ocasionam o desenvolvimento de doenças agudas e crônicas. Estes acometimentos são decorrentes do estresse persistente e depressões do sistema imune, que ocasionam quadros de hiperglicemia, diabetes, cefaleia, artralgia e complicações da hipertensão arterial sistêmica, que acaba por se apresentar como problema mais frequente, associado à esta sobrecarga e chegando a desencadear eventos cerebrovasculares e síndromes coronarianas.

Quanto às afetações emocionais, infligem aos cuidadores de pessoas com DA inúmeros problemas, decorrentes, em grande parte, da mudança do estilo de vida, que se impõe a estas pessoas, intensificada pelos altos níveis de estresse e subvalorização das suas necessidades, em detrimento das necessidades do doente de Alzheimer. Este fator leva ao desencadeamento de quadros de ansiedade, depressão, sentimento de impotência e frustração por parte daqueles de promovem esses cuidados.

Percebe-se a necessidade de um maior engajamento de terceiros, sendo de fora ou do seio familiar, e o acompanhamento dos cuidadores de pessoas acometidos de Alzheimer, pois a saúde destes está diretamente ligada com os cuidados que serão dispensados aos pacientes com DA. É necessária a ampliação e a efetivação de redes de apoio, tanto relacionadas ao suporte em saúde quanto a uma rede de apoio familiar, no sentido de compartilhamento das atribuições e cuidados que serão destinados ao doente, como rodas de conversa, partilha de tarefas e, até, um acompanhamento mais próximo das equipes de saúde, a fim de evitar o processo de adoecimento do próprio cuidador.

Por se tratar de um estudo de revisão integrativa, o presente trabalho apresenta algumas limitações, como a coleta dos dados de forma secundária, sem o acompanhamento real dos cuidadores de pacientes com demência de Alzheimer, de modo que não foi possível detectar a intensidade das afecções físicas e emocionais decorrentes do processo de cuidar. Por conta disso, é interessante que novos estudos, com abordagens qualitativas e randomizadas, busquem identificar, de forma íntima, quais as alterações físicas e emocionais que alteram a saúde no cuidador no processo de cuidar dos pacientes com Alzheimer, visando uma melhor abordagem desse público e garantia do seu bem-estar e qualidade de vida, mesmo diante desta situação complexa.

\section{Referências}

Aboulafia-Brakha, T., Suchecki, D., Gouveia-Paulino, F., Nitrini, R., \& Ptak, R. (2014). Cognitive-behavioural group therapy improves a psychophysiological marker of stress in caregivers of patients with Alzheimer's disease. Aging \& Mental Health, 18(6), 801-808. https://pubmed.ncbi.nlm.nih.gov/24499394/. 
Andrade, A. M. E. (2012). Factores de riesgo de carga en cuidadores informales de adultos mayores con demencia. Revista Cubana de Salud Pública, 38(2), 108-128. http://scielo.sld.cu/scielo.php?pid=S0864-34662012000300006\&script=sci_abstract.

Araújo, C. L. O. de., Oliveira, J. F., \& Pereira, J. M. (2012). Perfil de cuidadores de idosos com Doença de Alzheimer. Revista Kairós Gerontologia, 15(2), 109-128. https://revistas.pucsp.br/index.php/kairos/article/viewFile/13109/9638.

Campos, C. R. F., Carvalho, T. R. de., Barham, E. J., Andrade, L. R. F. de., \& Giannini, A. S. (2019). Entender e envolver: avaliando dois objetivos de um programa para cuidadores de idosos com Alzheimer. Psico, 50(1), 1-12. https://revistaseletronicas.pucrs.br/ojs/index.php/revistapsico/article/view/29444.

Cesário, V. A. C., Leal, M. C. C., Marques, A. P. O., \& Claudino, K. A. (2017). Estresse e qualidade de vida do cuidador familiar de idoso portador da doença de Alzheimer. Saúde em debate, 41(112), 171-182. https://www.scielo.br/j/sdeb/a/YPgdVBvzrhMy7XKcxXNj9Hn/abstract/?lang=pt.

Charlesworth, G., Burnell, K., Crellin, N., Hoare, Z., Hoe, J., \& Knapp, M. et al. (2016). Peer support and reminiscence therapy for people with dementia and their family carers: a factorial pragmatic randomized trial. Journal of Neurology, Neurosurgery \& Psychiatry, 87(11), 1218-1228. https://pubmed.ncbi.nlm.nih.gov/27521377/.

Cheng, S., Lau, R.W., Mak, E. P., Ng, N., Lam, L., \& Fung, H. et al. (2012). A benefit-finding intervention for family caregivers of persons with Alzheimer disease: study protocol of a randomized controlled trial. Trials, 13(1), 1-10. https://pubmed.ncbi.nlm.nih.gov/22747914/.

Coelho, G. G., Teixeira, P. S., Novak, J. C., \& Galhardi, M. (2015). Os impactos na saúde do cuidador familiar de pessoas com doença de Alzheimer: uma revisão bibliográfica. Revista Ciência e Estudos Acadêmicos de https://periodicos.unemat.br/index.php/revistamedicina/article/view/411/848.

Córdoba, A. M. C., \& Aparicio, M. J. G. (2014). Efectos de cuidar personas con Alzheimer: um estudio sobre cuidadores formales e informales. Pensamiento Psicológico, 12(1), 149-167. https://www.redalyc.org/pdf/801/80131179010.pdf.

Farina, N., Page, T., Daley, S., Brown, A., Bowling, A., \& Basset, T. et al. (2017). Factors associated with the quality of life of family carers of people with dementia: A systematic review. Alzheimer's \& Dementia, 13(5), 572-581. https://pubmed.ncbi.nlm.nih.gov/28167069/.

Gonçalves-Pereira, M., \& Sampaio, D. (2011). Psicoeducação familiar na demência: da clínica à saúde pública. Revista Portuguesa de Saúde Pública, 29(1), 3-10. https://www.elsevier.es/en-revista-revista-portuguesa-saude-publica-323-articulo-psicoeducacao-familiar-na-demencia-da-clinica-a-saudeS0870902511700025

Gratao, A. C. M., Talmelli, L., Figueiredo, L., Rosset, I., Freitas, C., \& Rodrigues, R. (2013). Dependência funcional de idosos e a sobrecarga do cuidador. Revista da Escola de Enfermagem da USP, 47(1), 137-144. https://www.scielo.br/j/reeusp/a/G6HxJWZYFRYYyvWFpCXjF3K/?lang=pt.

Gratao, A. C. M., Vale, F., Roriz-Cruz, M., Haas, V., Lange, C., Talmelli, L., \& Rodrigues, R. (2010). The demands of Family caregivers of elderly individuals with dementia. Revista da Escola de Enfermagem da USP, 44(4), 873-880. https://www.scielo.br/j/reeusp/a/bPCDhLrt43tZmLVjwv6SSns/abstract/?lang=en.

Kishita, N., Hammond, L., Dietrich, C., \& Mioshi, E. (2018). Which interventions work for dementia family carers? An updated systematic review of randomized controlled trials of carer interventions. International Psychogeriatrics, 30(11), 1679-1696. https://pubmed.ncbi.nlm.nih.gov/30017008/.

Kucmanski, L., Zanevicz, L., Geremia, D., Madureira, V., Silva, T., \& Souza, S. (2016). Doença de Alzheimer: desafios enfrentados pelo cuidador no cotidiano familiar. Revista Brasileira de Geriatria e e $1922-1029$. https://www.scielo.br/j/rbgg/a/9rNYm9FRGdnJxgM5rf3cMWM/?lang=pt.

Leite, B. S., Camacho, A. C. L. F., Joaquim, F. L., Gurgel, J. L., Lima, T. R., \& Queiroz, R. S. de. (2017). A vulnerabilidade dos cuidadores de idosos com demência: estudo descritivo transversal. Revista Brasileira de 714-720. https://www.scielo.br/j/reben/a/chtJwWjyZCvDth9b8Tm9VBr/?lang=pt\&format=pdf.

Manzini, C. S. S., \& Vale, F. A. C. do. Emotional Disorders evidenced by Family caregivers of older people with Alzheimer's disease. Dementia \& Neuropsychologia, 14(1), 56-61. https://www.scielo.br/j/dn/a/CPW4BHKYjV7RsW793TRq6Zz/?lang=en.

Marins, A. M. F., Hansel, C. G., \& Silva, J. da. (2016). Mudanças de comportamento em idosos com doença de Alzheimer e sobrecarga para o cuidador. Escola Anna Nery - Revista de Enfermagem, 20(2), 352-356. https://www.scielo.br/j/ean/a/GDDrj9PsLd79QjNSfrc9NHR/?lang=pt\&format=pdf.

Mattos, E. B. T., \& Kovács, M. J. Doença de Alzheimer: a experiência única de cuidadores familiares. Psicologia USP, 38(4), 294-300. https://www.revistas.usp.br/psicousp/article/view/168601.

Moore, R., Chattillion, E., Ceglowski, J., Ho, J., Von Kanel, R., \& Mills, P. et al. (2013). A randomized clinical trial of Behavioral activation (BA) therapy for improving psychological and physical health in dementia caregivers: Results of the Pleasant Events Program (PEP). Behaviour Research and Therapy, 51(10), 623-632. https://www.ncbi.nlm.nih.gov/pmc/articles/PMC3774137/.

Perdomo, M. P., Poey, M. C., \& Oca, B. L. O. M. (2012). Variables asociadas con la depresión y la sobrecarga que experimentan los cuidadores principales de los ancianos con Alzheimer. Revista Cubana de Enfermería, 28(4), 509-520. http://scielo.sld.cu/scielo.php?script=sci_arttext\&pid=S086403192012000400007

Perdomo, M. P., \& Rodríguez, J. J. L. (2010). Características sociodemográficas y nivel de sobrecarga de cuidadores de ancianos con Enfermedad de Alzheimer. Revista Cubana de Enfermería, 26(3), 110-122. http://scielo.sld.cu/scielo.php?script=sci_arttext\&pid=S0864-03192010000300003.

Petrovic, M., \& Gaggiolo, A. (2020). Digital Mental Health Tools for caregivers of Older Adults. Frontiers in Public Health, 8(126), 1-10. https://pubmed.ncbi.nlm.nih.gov/32411643/.

Ponce, C. C., Ordonez, T. N., Lima-Silva, T. B., Santos, G. D. D., Viola, D. F., \& Nunes, P. V. et al. (2011). Effects of a psychoeducational intervention in family caregivers of people with Alzheimer's disease. Dementia \& Neuropsychologia, 5(3), 226-237. https://www.scielo.br/j/dn/a/nc5rqwFzPQ6JD5GQzqpGq4z/abstract/?lang=en. 
Research, Society and Development, v. 10, n. 8, e1410816990, 2021

(CC BY 4.0) | ISSN 2525-3409 | DOI: http://dx.doi.org/10.33448/rsd-v10i8.16990

Santos, A. A. dos., \& Pavarini, S. C. I. (2012). Funcionalidade familiar de idosos com alterações cognitivas: a percepção do cuidador. Revista da Escola de Enfermagem da USP, 46(5), 1141-1147. https://www.scielo.br/j/reeusp/a/Z4VzDCmhk3NVWQ954RxLCBP/abstract/?lang=pt.

Silva, C. F. da., Passos, V. M. de. A., \& Barreto, S. M. (2012). Frequência e repercussão da sobrecaga de cuidadoras familiares de idosos com demência. Revista Brasileira de Geriatria e Gerontologia, 15(4), 707-731. https://www.scielo.br/j/rbgg/a/7Ydj3ySk8N4Fgcng74DBpzC/abstract/?lang=pt.

Souza, M. T. de., Silva, M. D. da., Carvalho, R. de. (2010). Revisão integrativa: o que é e como fazer. Einstein, 8(1), 102-106. https://www.scielo.br/j/eins/a/ZQTBkVJZqcWrTT34cXLjtBx/?lang=pt\&format=pdf.

Terum, T. M., Andersen, J. R., Rongve, A., Aarsland, D., Svendsboe, E. J., \& Testad, I. (2017). The relationship of specific items on the Neuropsychiatric Inventory to caregiver burden in dementia: a systematic review. International Journal of geriatric psychiatry, 32(7), 703-717. https://pubmed.ncbi.nlm.nih.gov/28317166/.

Vizzachi, B. A., Daspett, C., Cruz, M. G. da. S., \& Horta, A. L. de. M. (2015). A dinâmica familiar diante da doença de Alzheimer em um de seus membros. Revista da Escola de Enfermagem da USP, 49(6), 933-938. https://www.scielo.br/j/reeusp/a/bj8Y9hQSPr9t97rhT36CRvS/?lang=pt\&format=pdf. 